\title{
GSOS for non-deterministic processes with quantitative aspects
}

\author{
Marino Miculan Marco Peressotti \\ Laboratory of Models and Applications of Distributed Systems, \\ Department of Mathematics and Computer Science, University of Udine, Italy
}

marino.miculan@uniud.it

marco.peressotti@uniud.it

\begin{abstract}
Recently, some general frameworks have been proposed as unifying theories for processes combining non-determinism with quantitative aspects (such as probabilistic or stochastically timed executions), aiming to provide general results and tools. This paper provides two contributions in this respect. First, we present a general GSOS specification format (and a corresponding notion of bisimulation) for non-deterministic processes with quantitative aspects. These specifications define labelled transition systems according to the ULTraS model, an extension of the usual LTSs where the transition relation associates any source state and transition label with state reachability weight functions (like, e.g., probability distributions). This format, hence called Weight Function SOS (WFSOS), covers many known systems and their bisimulations (e.g. PEPA, TIPP, PCSP) and GSOS formats (e.g. GSOS, Weighted GSOS, Segala-GSOS, among others).

The second contribution is a characterization of these systems as coalgebras of a class of functors, parametric on the weight structure. This result allows us to prove soundness of the WFSOS specification format, and that bisimilarities induced by these specifications are always congruences.
\end{abstract}

\section{Introduction}

Process calculi and labelled transition systems have proved very successful for modelling and analysing concurrent, non-deterministic systems. This success has led to many extensions dealing with quantitative aspects, by adding further informations to the transition relation (e.g. probability rates or stochastic rates); see [ [ $\left.\begin{array}{l|l||l|l|l|l|l}3 & 4 & 11 & 12 & 16 & 24\end{array}\right]$ among others. These calculi have proved very effective in modelling and reasoning about systems, like performance analysis of computer networks, model checking of time-critical systems, simulation of biological systems, probabilistic analysis of security and safety properties, etc.

Each of these calculi is tailored to a specific quantitative aspect and for each of them we have to develop a quite complex theory almost from scratch. This is a daunting and error-prone task, as it embraces the definition of syntax, semantics, labelled transition rules, various behavioural equivalences, logics, proof systems; the proof of important properties like congruence of behavioural equivalences; the development of algorithms and tools for simulations, model checking, etc. This situation would naturally benefit from general frameworks for LTS with quantitative aspects, i.e., mathematical metamodels offering general methodologies, results, and tools, which can be uniformly instantiated to a wide range of specific calculi and models. In recent years, some of these theories have been proposed; we mention Functional Transition Systems (FuTS) [18], weighted labelled transition systems (WLTSs) [16. 28], and in particular Uniform Labelled Transition Systems (ULTraS), introduced by Bernardo, De Nicola and Loreti specifically as "a uniform setting for modelling non-deterministic, probabilistic, stochastic or mixed processes and their behavioural equivalences" [3].

A common feature of most of these meta-models is that their labelled transition relations do not yield states (i.e., a process), but some mathematical object representing quantitative informations about "how"

N. Bertand and L. Bortolussi (Eds.): QAPL 2014

EPTCS 154, 2014, pp. 17-33 doi 10.4204/EPTCS.154.2 (c) M. Miculan \& M. Peressotti

This work is licensed under the Creative Commons Attribution License. 
each state can be reached. In particular, transitions in ULTraS systems have the form $P \stackrel{a}{\rightarrow} \rho$ where $\rho$ is a state reachability weight function, i.e., a function assigning a weight to each possible state. By suitably choosing the set of weights, and how these functions combine, we can recover ordinary non-deterministic LTSs, probabilistic transition systems, stochastic transition systems, etc. As convincingly argued in [3], the use of weight functions in place of plain processes simplifies the combination of non-determinism with quantitative aspects, like in the case of EMPA or PEPA. Moreover, it paves the way for general definitions and results, an important example being the notion of $\mathscr{M}$-bisimulation [3].

Albeit quite effective, these meta-models are at their dawn, with many results and techniques still to be developed. An important example of these missing notions is a specification format, like the wellknown GSOS format for non-deterministic labelled transition systems. These formats are very useful in practice, because they can be used for ensuring important properties of the system; in particular, the bisimulations induced by systems in these formats is guaranteed to be a congruence (which is crucial for compositional reasoning). From a more foundational point of view, these frameworks would benefit from a categorical characterization in the theory of coalgebras and bialgebras: this would allow a crossfertilizing exchange of definitions, notions and techniques with similar contexts and theories.

In this paper, we provide two main contributions in this respect. First, we present a GSOS-style format, called Weight Function SOS (WFSOS), for the specifications of non-deterministic systems with quantitative aspects. The judgement derived by rules in this style is of the form $P \stackrel{a}{\longrightarrow} \psi$, where $P$ is a process and $\psi$ is a weight function term. These terms describe weight functions, by means of an interpretation. A specification given in this format defines a ULTraS (although we could work also in other frameworks, such as FuTS). By choosing the set of weights, the language of weight function terms and their interpretation, we can readily capture many quantitative notions (probabilistic, stochastic, etc.), and different kinds of non-deterministic interactions, covering models like PEPA, TIPP, PCSP, EMPA, among others. Moreover, the WFSOS format supports a general definition of (strong) bisimulation, which can be readily instantiated to the various specific systems.

The second contribution aims to be more fundamental. We provide a general categorical presentation of these non-deterministic systems with quantitative aspects. Namely, we prove that ULTraS systems are in one-to-one correspondence with coalgebras of a precise class of functors, parametric on the underlying weight structure. Using this characterization we show that each WFSOS specification yields a $\lambda$-distributive law (i.e. a natural transformation of a specific shape) for these functors. Thus, taking advantage of Turi-Plotkin's bialgebraic framework, we can prove that the bisimulation induced by a WFSOS is always a congruence, thus allowing for compositional reasoning in quantitative settings.

The developments closest to ours are Klin's Weighted GSOS, a rule format for WLTS [16], and Bartel's Segala GSOS, a rule format for Segala systems [2, §5.3]. Both Segala systems and WLTS are subsumed by ULTraS (in fact, WLTSs correspond to "deterministic" ULTraSs, where a process is associated to exactly one weight function for each label), and as we will show, WFSOS subsumes WGSOS and Segala GSOS. On a different direction, in [9] De Nicola et al. provide a "meta-calculus" for describing various stochastic systems and their semantics as FuTS, showing that in several cases behavioural equivalences are congruences. This interesting approach is complementary to ours, since it provides some "syntactic-semantic basic blocks" to be assembled, instead of a general rule format.

The rest of the paper is structured as follows. In Section 2 we recall Uniform Labelled Transition Systems, and their bisimulation. In Section 3 we introduce the Weight Function SOS specification format for the syntactic presentation of ULTraSs. In Section 4 we apply this format to PEPA, and show that it subsumes other GSOS formats like WGSOS and Segala GSOS. The categorical presentation of ULTraS and WFSOS, with the result that bisimilarity is a congruence, is given in Section 5 . Final remarks and directions for future work are in Section 6 . 


\section{Uniform Labelled Transition Systems}

In this Section we recall and elaborate the definition of ULTraSs and describe their (coalgebraically derived) bisimulation, offering a comparison with Bernardo's (more general) notion of $\mathscr{M}$-bisimulation presented in [3]. Although we focus on the specific framework of ULTraS, results and methodologies described in this paper can be ported to other formats with similar features (like FuTS [18]), and more generally to a wide range of systems combining different computational aspects in different ways.

Uniform Labelled Transition Systems ULTraS are (non-deterministic) labelled transition systems whose transitions lead to state reachability weight functions, i.e. functions representing quantitative informations about "how" each state can be reached. Examples of weight functions include probability distributions, resource consumption levels, or stochastic rates. Under this light, ULTraS can be thought as "generalized" Segala systems [27] (which stratify non-determinism over probabilism). Following the parallel with Segala systems, ULTraS transitions can be pictured as being composed by two steps:

$$
x \stackrel{a}{\longrightarrow} \rho \stackrel{w}{\longrightarrow} y
$$

where the first is a labelled non-deterministic (sub)transition and the second is a weighted one; from this perspective the weight function plays the rôle of the "hidden intermediate state".

Akin to Weighted Labelled Transition Systems (WLTS) [10, 16, 28], weights are drawn from a fixed set endowed with a commutative monoid structure, where the unit is meant to be assigned to disabled transitions (i.e. those yielding unreachable states) and the monoidal addition is used to compositionally weight sets of transitions given by non-determinism.

Definition 1 (ULTraS). Given a commutative monoid $\mathfrak{W}=(W,+, 0)$, a (WJ-weighted) Uniform Labelled Transition System (WU-ULTraS) is a triple $(X, A, \rightarrow)$ where:

- $X$ is a set of states (processes);

- A is a set of labels (actions);

- $\rightarrow \subseteq X \times A \times[X \rightarrow W]$ is a transition relation and $[X \rightarrow W]$ is the set of weight functions.

Monoidal addition does not play any rôle in the above definition ${ }^{1}$ but it is crucial to the notion of bisimulation by uniformly providing a compositional way to weight sets of outgoing transitions (e.g. stochastic or probabilistic bisimulations). Because total weights are defined by summation, some guarantees on the cardinality of these sets are needed:

Definition 2 (Image boundedness). Let $\kappa$ and $\kappa^{\prime}$ be ordinals and let $\mathfrak{W}=(W,+, 0)$ be a commutative monoid. A weight function $\rho$ is $\kappa$-supported iff $|\{x \in \operatorname{dom}(\rho) \mid \rho(x) \neq 0\}|<\kappa$. A $\mathfrak{W}$-ULTraS $(X, A, \rightarrow)$ is image $\left(\kappa, \kappa^{\prime}\right)$-bounded iff for any state $x \in X$ and label $a \in A$ the set $\{\rho \mid x \stackrel{a}{\triangleright} \rho\}$ has cardinality less than $\kappa$ and contains only $\kappa^{\prime}$-supported functions. (We shall drop $\kappa^{\prime}$ when it is equal to $\kappa$.)

The notion of image boundedness just introduced guarantees that the branching transitions do not exceed the expressive power of summation of the underlying monoid in the sense that, if sum is defined for any family of cardinality lesser than $\kappa$, then for any state in a $\kappa$-bounded system the total weights for sets of outgoing transitions are always defined. Henceforth, for the sake of simplicity, we will restrict ourselves to image finite systems (i.e. $\omega$-bounded), but the development can be generalized throughout.

\footnotetext{
${ }^{1}$ In $[3]$ weights are assumed to be a pointed set. Actually, a partial order is assumed, but the ordering is not crucial to the basic notion of ULTraS as it is only required by some equivalences considered in that paper.
} 
Notation. Let $\mathfrak{W}=(W,+, 0)$ be a commutative monoid. For each function $\rho: X \rightarrow W$ the set $\{x \mid \rho(x) \neq$ $0\}$ is called support of $\rho$ (written $\lceil\rho\rceil$ ). For any set $X$ let $\mathscr{P}_{f} X$ denote the finite powerset of $X$ and let $\mathscr{F}_{\mathfrak{W}} X$ be the set $\left\{\rho: X \rightarrow W \mid\lceil\rho\rceil \in \mathscr{P}_{f} X\right\}$ of finitely supported weight functions. For each $\rho \in \mathscr{F}_{\mathfrak{W}} X$ let $\rho(Y)=\sum_{x \in Y} \rho(x)$, for any $Y \subseteq X$ and define the total weight of $\rho$ as $\left.\llbracket \rho\right\rceil \triangleq \rho(X)=\rho(\lceil\rho\rceil)$.

We give now the definition of bisimulation for ULTraS, which arises directly from the coalgebraic understanding of ULTraS which we will introduce in Section 5 .

Definition 3 (Bisimulation). Let $\left(X, A, \rightarrow_{X}\right)$ and $\left(Y, A, \rightarrow_{Y}\right)$ be two image finite $\mathfrak{W}-U L T r a S$. An equivalence relation $R$ between $X$ and $Y$ is a bisimulation if, and only if, for each pair of states $x \in X$ and $y \in Y$, $(x, y) \in R$ implies that for each label $a \in A$ the following holds:

- if $x \stackrel{a}{\rightarrow}{ }_{X} \varphi$ then there exists $y \stackrel{a}{\rightarrow} Y \psi$ s.t. for each equivalence class $C$ of $R: \varphi(C)=\psi(C)$;

- if $y \stackrel{a}{\triangleright_{Y}} \psi$ then there exists $x \stackrel{a}{D_{X}} \varphi$ s.t. for each equivalence class $C$ of $R: \psi(C)=\varphi(C)$.

Processes $x$ and $y$ are said to be bisimilar if there exists a bisimulation $R$ such that $(x, y) \in R$.

Likewise ULTraSs can be though as stacking non-determinism over other computational behaviour, Definition 3 stratifies bisimulation for non-deterministic labelled transition system over bisimulation for systems expressible as labelled transition systems weighted over commutative monoids such as stochastic or probabilistic systems. In fact, two processes $x$ and $y$ are related by some bisimulation if, and only if, whether one reaches a weight function via a non-deterministic labelled transition, the other can reach an equivalent function via a transition with the same label where the reached functions are equivalent in the sense that they assign the same total weight to the same classes of states in the relation. For instance, in the case of weights being probabilities, functions are considered equivalent only when they agree on the probabilities assigned to each class of states which is precisely the intuition behind probabilistic bisimulation [17]. More examples will be discussed below.

Constrained ULTraS Sometimes, the ULTraSs given by some monoid are too general; for instance, fully-stochastic systems such as (labelled) CTMCs are a strict subclass of ULTraSs weighted over the monoid of non-negative real numbers $\left(\mathbb{R}_{0}^{+},+, 0\right)$, where weights express rates of exponentially distributed continuous time transitions. In the case of fully-stochastic systems, for each label, each state is associated with precisely one weight function. This kind of "deterministic" ULTraSs are called functional in [3], since the transition relation is functional, and correspond precisely to WLTSs (cf. [10, 16, 28]). These are a well-known family of systems (especially their automata counterpart) and have an established coalgebraic understanding as long as a (coalgebraically derived) notion of weighted bisimulation which are shown to subsume several known kinds of systems such as non-deterministic, (fully) stochastic, generative and reactive probabilistic [16]. Moreover, Definition 3 coincides with weighted bisimulation [16, Def. 4] on functional ULTraSs/WLTSs over the same monoid and hence Definition 3 covers every system expressible in the framework of WLTS.

Proposition 1. Let $\mathfrak{W}$ be a commutative monoid and $(X, A, \rightarrow)$ be a $\mathfrak{W}-L T S$ seen as a functional ULTraS on $\mathfrak{W}$. Every bisimulation relation on $\rightarrow$ is a $\mathfrak{W}$-weighted bisimulation and vice versa.

Another constraint arises in the case of probabilistic systems, i.e., weight functions are probability distribution. Since addition is not a closed operation in the unit interval $[0,1]$, there is no monoid $\mathfrak{W}$ such that every weight function on it is also a probability distributions. We could relax Definition 1 to allow commutative partial monoids $\mathrm{s}^{2}$ such as the weight structure of probabilities $\left.([0,1],+, 0)\right)$. Unfortunately,

\footnotetext{
${ }^{2}$ A commutative partial monoid is a set endowed with a partial binary operation with a unit such that it is associative and commutative, where it is defined, and always defined on its unit.
} 
not every weight function on $[0,1]$ is a probability distribution. In fact, probabilistic systems (among others) can be recovered as ULTraSs over the $\left(\mathbb{R}_{0}^{+},+, 0\right)$ (i.e. the free completion of $\left.([0,1],+, 0)\right)$ and subject to suitable constraints. For instance, Segala systems [27] are precisely the strict subclass of $\mathbb{R}_{0}^{+}$-ULTraS such that every weight function $\rho$ in their transition relation is a probability distribution i.e. $\llbracket \rho \rrbracket=1$. Moreover, bisimulation is preserved by constraints. For instance, bisimulations on the above class of (constrained) ULTraS are Segala's (strong) bisimulations (cf. [27, Def. 13]), and vice versa.

Proposition 2. Let $(X, A, \rightarrow)$ be an image finite Segala-system seen as a ULTraS on $\left(\mathbb{R}_{0}^{+},+, 0\right)$. Every bisimulation relation on $\rightarrow$ is a strong bisimulation in the sense of [27. Def. 13] and vice versa.

A similar result holds for reactive and generative (or fully) probabilistic systems and their bisimulations. In fact, these are functional ULTraS with weight functions in $\mathscr{F}_{\mathbb{R}_{+}^{0}}$ and subject to constraints $\forall x \sum_{a \in A, y \in X} \mathrm{P}(x, a, y) \in\{0,1\}$ and $\forall x \in X, a \in A \sum_{y \in X} \rho(x, a, y) \in\{0,1\}$ respectively.

About $\mathscr{M}$-bisimulation Bernardo et al. defined a notion of bisimulation for ULTraS [3, Def. 3.3], parametrized by a function $\mathscr{M}$ which is used to weight sets of transitions. Notably, $\mathscr{M}$ 's codomain may be not the same of that used for weight functions in the transition relation. This offers an extra degree of freedom with respect to Definition 3 . We recall the relevant definitions. $3^{3}$

Definition 4 (M-function). Let $(M, \perp)$ be a pointed set and $(X, A, \rightarrow)$ be a W-ULTraS. A function $\mathscr{M}: X \times A \times \mathscr{P} X \rightarrow M$ is an $M$-function for $(X, A, \rightarrow)$ iff

- for all $x \in X, a \in A$ and $C \in \mathscr{P} X, \mathscr{M}(x, a, C)=\perp$ whenever $\rho(C)=0$ for every $x \stackrel{a}{\longrightarrow} \rho$ or there is no $\rho$ at all;

- for all $x, y \in X, a \in A$ and $C_{1}, C_{2} \in \mathscr{P} X, \mathscr{M}\left(x, a, C_{1}\right)=\mathscr{M}\left(y, a, C_{1}\right)$ and $\mathscr{M}\left(x, a, C_{2}\right)=\mathscr{M}\left(y, a, C_{2}\right)$ implies that $\mathscr{M}\left(x, a, C_{1} \cup C_{2}\right)=\mathscr{M}\left(y, a, C_{1} \cup C_{2}\right)$.

Definition 5 ( $\mathscr{M}$-bisimulation [3]). Let $\mathscr{M}$ be an $M$-function for $(X, A, \rightarrow)$. A relation $R \subseteq X \times X$ is a $\mathscr{M}$-bisimulation for $\rightarrow$ iff for each pair $(x, y) \in R$, label $a \in A$, and class $C \in(X / R)$ the following holds:

$$
\mathscr{M}(x, a, C)=\mathscr{M}(y, a, C) .
$$

The notion of $\mathscr{M}$-bisimulation is somehow more general than Definition 3 since (sets of) transitions are not necessarily weighted in the same structures. For instance, stochastic rates can be considered up-to a suitable tolerance in order to account for experimental measurement errors in the model.

A further distinction arises from the fact that ULTraSs came with two distinct way of "terminating". A state can be seen as "terminated" either when its outgoing transitions are always the constantly zero function, or when it has no transitions at all. In the first case, the state has still associated an outcome, saying that no further state is reachable; we call these states terminal. In the second case, the LTS does not even tell us that the state cannot reach any further state; in fact, there is no "meaning" associated to the state. In this case, we say that the state is stuck $4^{4}$ The bisimulation given in Definition 3 keeps these two terminations as different (i.e., they are not bisimilar), whereas $\mathscr{M}$-bisimulation does not make this distinction (cf. [3, Def. 3.2] or, for a concrete example based on Segala systems, [3, Def. 7.2]). Finally, the two notions differ on the quantification over equivalence classes: in the case of Definition 3 quantification depends on the non-deterministic step whereas in the case of $\mathscr{M}$-bisimulation it does not.

\footnotetext{
${ }^{3}$ In the original presentation, $\mathscr{M}$ is required to consistently weight also sequences of transitions, in order to cover also trace equivalence; because this Section focuses on bisimulations only, this information will be omitted.

${ }^{4}$ To make a comparison with sequential programs, a terminal state is when the execution reaches the end of the program; a stuck state is when we are at executing an instruction whose meaning is not defined.
} 
Under some mild assumptions, the two notions agree. In particular, let us restrict to systems with just one of the two terminations for each action $a$-i.e. for pair of states $x, y,\{\rho \mid x \stackrel{a}{\rightarrow} \rho\}=\emptyset$ implies $\lambda z .0 \notin\{\rho \mid y \stackrel{a}{\rightarrow} \rho\}$, and, symmetrically, $\lambda z .0 \in\{\rho \mid x \stackrel{a}{\rightarrow} \rho\}$ implies $\{\rho \mid y \stackrel{a}{\rightarrow} \rho\} \neq \emptyset$. Then, for suitable choices of $\mathscr{M}, \mathscr{M}$-bisimulation agrees with the notion given in Definition 3 (on one system).

Proposition 3. Let $(X, A, \rightarrow)$ be a $\mathfrak{W}-U L T r a S$ such that for any label, each pair of states has at most one kind of termination. Every bisimulation $R$ is also an $\mathscr{M}$-bisimulation for $\mathscr{M}(x, a, C)=\left\{[\rho]_{\equiv_{R}} \mid\right.$ $x \stackrel{a}{\triangleright} \rho \wedge \rho(C) \neq 0\} \cup \varnothing$ where $(M, \perp)=\left(\mathscr{P}_{f}\left(\mathscr{F}_{\mathfrak{W}} X / \equiv_{R}\right), \varnothing\right), \varphi \equiv_{R} \psi \stackrel{\triangle}{\Longleftrightarrow} \forall C \in X / R . \varphi(C)=\psi(C)$, and $\varnothing=\left\{[\lambda z .0]_{\equiv_{R}}\right\}$.

\section{WFSOS: A GSOS format for ULTraSs}

In this section we introduce the Weight Function SOS specification format for the syntactic presentation of ULTraSs. As it will be proven in Section 5.3, bisimilarity for systems given in this format is guaranteed to be a congruence with respect to the signature used for representing processes.

The format is parametric in the weight monoid $\mathfrak{W}$ and, as usual, in the process signature $\Sigma$ defining the syntax of system processes. In contrast with "classic" GSOS formats [15], targets of rules are not processes but terms whose syntax is given by a different signature, called the weight signature. This syntax can be thought as an "intermediate language" for representing weight functions along the line of viewing ULTraSs as stratified (or staged) systems. An early example of this approach can be found in [1], where targets are terms representing measures over the continuous state space. Earlier steps in this direction can be found e.g. in Bartels' GSOS format for Segala systems (cf. Section 4 and [2, §5.3]) or in [3,8] where targets are presented by meta-expressions.

Definition 6 (WFSOS Rule). Let $\mathfrak{W}$ be a commutative monoid and $A$ a set of labels. Let $\Sigma$ and $\Theta$ be the process signature and the weight signature respectively. A WFSOS rule over them is a rule of the form:

$$
\begin{aligned}
& \left\{x_{i} \stackrel{a}{\triangleright} \varphi_{i j}^{a}\right\}_{\substack{1 \leq i \leq n, a \in A_{i} \\
1 \leq j \leq m_{i}^{a}}}, \quad\left\{x_{i} \stackrel{b}{p}\right\}_{\substack{1 \leq i \leq n, b \in B_{i}}} \quad\left\{\llbracket \varphi_{i_{k} j_{k}}^{a_{k}} \|=w_{k}\right\}_{1 \leq k \leq p} \quad\left\{\left\lceil\varphi_{i_{k} j_{k}}^{a_{k}}\right\rceil \ni y_{k}\right\}_{1 \leq k \leq q} \\
& \mathrm{f}\left(x_{1}, \ldots, x_{n}\right) \stackrel{c}{\longrightarrow} \psi
\end{aligned}
$$

where:

- $\mathrm{f}$ is an $n$-ary symbol from $\Sigma$;

- $X=\left\{x_{i} \mid 1 \leq i \leq n\right\}, Y=\left\{y_{k} \mid 1 \leq k \leq q\right\}$ are sets of pairwise distinct process variables;

- $\Phi=\left\{\varphi_{i j}^{a} \mid 1 \leq i \leq n, a \in A_{i}, 1 \leq j \leq m_{i}^{a}\right\}$ is a set of pairwise distinct weight function variables;

- $\left\{w_{k} \in \mathfrak{W} \mid 1 \leq k \leq p\right\}$ are weight constants s.t. $w_{k} \neq 0$ for $1 \leq k \leq q$;

- $a, b, c \in A$ are labels and $A_{i} \cap B_{i}=\emptyset$ for $1 \leq i \leq n$;

- $\psi$ is a weight term for the signature $\Theta$ such that $\operatorname{var}(\psi) \subseteq X \cup Y \cup \Phi$;

- if $q>0$ then $\mathfrak{W}$ must have the zerosumfree property (i.e. $w+w^{\prime}=0 \Longrightarrow w=w^{\prime}=0$ ).

A rule like above is triggered by a tuple $\left\langle C_{1}, \ldots, C_{n}\right\rangle$ of enabled labels and by a tuple of weights $\left\langle v_{1}, \ldots, v_{p}\right\rangle$ if, and only if, $A_{i}=C_{i} \subseteq A$ and $w_{k}=v_{k}$ for $1 \leq i \leq n$ and $1 \leq k \leq q$.

Intuitively, the four families of premises can be grouped in two kinds: the first two families correspond to the non-deterministic (and labelled) behaviour, whereas the other two correspond to the weighting behaviour of quantitative aspects. The former are precisely the premises of GSOS rules for LTSs (up-to targets being functions); and describe the possibility to perform some labelled transitions. The 
latter are inspired by Bartels' Segala GSOS [2, §5.3] and Klin's WGSOS [16] formats; a premise like $\| \varphi \rrbracket=w$ constrains the variable $\varphi$ to those functions whose total weight is exactly the constant $w$; a premise like $\lceil\varphi\rceil \ni y$ binds the process variable $y$ to those elements being assigned a non-zero weigh $5^{5}$ (i.e. reachable); clearly, premises of the latter kind assume that the support of $\varphi$ is not empty and hence $w_{k}$ is forced to be non-zero for every $1 \leq k \leq q$. Likewise Segala GSOS (but not WGSOS), there are no variables denoting the weight of $y_{k}$, but this information can be readily extracted from $\varphi_{i_{k} j_{k}}^{a_{k}}$, e.g. by some operator from $\Theta$.

Targets of transitions defined by these rules are terms generated from the signature $\Theta$. In order to characterize transition relations for ULTraSs, we need to evaluate these terms to weight functions. This is obtained by adding an interpretation for weight terms, besides a set of rules in the above format.

Before defining interpretations and specifications, we need to introduce some notations. For a signature $S$ and a set $X$ of variable symbols, let $T^{S} X$ denote the set of terms freely generated by $S$ over the variables $X$ (in the following, $S$ will be either $\Sigma$ or $\Theta$ ). A substitution for symbols in $X$ is any function $\sigma: X \rightarrow Y$; its action extends to terms defining the function $T^{S}(\sigma): T^{S} X \rightarrow T^{S} Y$ (i.e. simultaneous substitution). When confusion seems unlikely we use the more evocative $\mathrm{t}[\sigma]$ instead of $T^{S}(\sigma)(\mathrm{t})$.

A variable substitution $\sigma: X \rightarrow Y$ induces also a function $\mathscr{F}_{\mathfrak{W}}(\sigma): \mathscr{F}_{\mathfrak{W}} X \rightarrow \mathscr{F}_{\mathfrak{W}} Y$, mapping (finitely supported) weight functions over $X$ to (finitely supported) weight functions over $Y$, as follows:

$$
\mathscr{F}_{\mathfrak{W}}(\sigma)(\rho) \triangleq \lambda y: Y . \sum_{x \in \sigma^{-1}(y)} \rho(x)
$$

where $\sigma^{-1}$ is the pre-image through the map $\sigma$. Consistently, we denote the action of $\sigma$ on $\rho$ by $\rho[\sigma]$.

Definition 7 (Interpretation). Let $\mathfrak{W}$ be a commutative monoid, $\Sigma$ and $\Theta$ be the process and the weight signature respectively. A weight term interpretation for them is a family of functions

$$
(-)_{X}: T^{\Theta}\left(X+\mathscr{F}_{\mathfrak{W}}(X)\right) \rightarrow \mathscr{F}_{\mathfrak{W}} T^{\Sigma}(X)
$$

indexed over sets of variable symbols, and respecting substitutions, i.e.:

$$
\forall \sigma: X \rightarrow Y, \psi \in T^{\Theta}(X):(\psi)_{X}[\sigma]=(\psi[\sigma])_{Y}
$$

We are ready to introduce the WFSOS specification format. Basically, this is a set of WFSOS rules, subject to some finiteness conditions to ensure image finiteness, together with an interpretation.

Definition 8 (WFSOS specification). Let $\mathfrak{W}$ be a commutative monoid, A a set of labels, $\Sigma$ and $\Theta$ be the process and the weight signature respectively. An image-finite WFSOS specification over $\mathfrak{W}, \Sigma, \Theta$ is a pair $\langle\mathscr{R},(-)\rangle$ where (-) is a weight term interpretation and $\mathscr{R}$ is a set of rules compliant with Definition 6 and such that only finitely many rules share the same operator in the source (f), the same label in the conclusion (c), and the same trigger $\left\langle A_{1}, \ldots, A_{n}, w_{1}, \ldots, w_{p}\right\rangle$.

We can now describe how an ULTraS is obtained from a WFSOS specification.

Definition 9 (Induced ULTraS). The ULTraS induced by an image-finite WFSOS specification $\langle\mathscr{R},(-)\rangle$ over $\mathfrak{W}, \Sigma, \Theta$ is the $\mathfrak{W}$-ULTraS $\left(T^{\Sigma} \emptyset, A, \rightarrow\right)$ where $\rightarrow$ is defined as the smallest subset of $T^{\Sigma} \emptyset \times A \times$ $\mathscr{F}_{\mathfrak{W}} T^{\Sigma} \emptyset$ being closed under the following condition.

Let $p=\mathrm{f}\left(p_{1}, \ldots, p_{n}\right) \in T^{\Sigma} \emptyset$. Since the ground $\Sigma$-terms $p_{i}$ are structurally smaller than $p$ assume that the set $\left\{\rho \mid p_{i} \stackrel{a}{\longrightarrow} \rho\right\}$ - and hence the trigger $\vec{A}=\left\langle A_{1}, \ldots, A_{n}\right\rangle, \vec{w}=\left\langle w_{1}, \ldots, w_{q}\right\rangle-$ is determined for

\footnotetext{
${ }^{5}$ Actually, premises like $\lceil\varphi\rceil \ni y$ can bind only elements assigned a weight $u$ s.t. $u+v \neq 0$ for any $v$, since the action of $\mathscr{F}_{\mathfrak{W}}$ on substitutions (1) can annihilate variables assigned to weights with an inverse; a conservative solution is to allow these premises only in presence of monoids with the zerosumfree property.
} 
every $i \in\{1, \ldots, n\}$ and $a \in A$. For any rule $R \in \mathscr{R}$ whose conclusion is in the form $\mathrm{f}\left(x_{1}, \ldots, x_{n}\right) \stackrel{c}{\longrightarrow} \psi$ and triggered by $\vec{A}, \vec{w}$ let $X, Y, \Phi$ be the set of process and weight function variables involved in $R$ as per Definition 6 . Then, for any substitution $\sigma: X \cup Y \rightarrow T^{\Sigma} \emptyset$ and map $\theta: \Phi \rightarrow \mathscr{F}_{\mathfrak{W}} T^{\Sigma} \emptyset$ such that:

1. $\sigma\left(x_{i}\right)=p_{i}$ for $x_{i} \in X$;

2. $\theta\left(\varphi_{i j}^{a}\right)=\rho$ for each premise $x_{i} \stackrel{a}{\longrightarrow} \varphi_{i j}^{a}$ and $\left\|\varphi_{i j}^{a}\right\|=w_{k}$ of $R$, and for any $\rho$ s.t. $p_{i} \stackrel{a}{\longrightarrow} \rho$ and $\| \rho \rrbracket=w_{k}$

3. $\sigma\left(y_{k}\right)=q$ for each premise $\left\lceil\varphi_{i_{k} j_{k}}^{a_{k}}\right\rceil \ni y_{k}$ of $R$, and for any $q \in T^{\Sigma} \emptyset$ s.t. $\theta\left(\varphi_{i_{k} j_{k}}^{a_{k}}\right)(q) \neq 0$;

there is $p \stackrel{c}{\longrightarrow} \rho$ where $\rho \triangleq(\psi[\theta])_{X \cup Y}[\sigma]$ is the instantiated interpretation of the target $\Theta$-term $\psi$.

The above definition is well-given since it is based on structural recursion over ground $\Sigma$-terms (i.e. the process $p$ in each triple $(p, a, \rho))$; in particular, terms have finite depth and only structurally smaller terms are used by the recursion (i.e. the assumption of $p_{i} \stackrel{a}{\rightarrow} \rho$ being defined for each $p_{i}$ in $\left.p=\mathrm{f}\left(p_{1}, \ldots, p_{n}\right)\right)$. Moreover, for any trigger, operator, and conclusion label only finitely many rules have to be considered.

Finally we are able to state the main adequacy result for the proposed format although the proof is postponed to Section 5.3, where we will take advantage of the bialgebraic framework.

Theorem 4 (Congruence). Behavioural equivalence on ULTraSs induced by a WFSOS specification is a congruence with respect to the process signature.

Remark 1 (Expressing interpretations). The definition of a weight term interpretation can be done in many ways, such as structural recursion or $\lambda$-iteration [1]. For instance, every family of maps:

$$
h_{X}: \Theta \mathscr{F}_{\mathfrak{W}} T^{\Sigma}(X) \rightarrow \mathscr{F}_{\mathfrak{W}} T^{\Sigma}(X) \quad b_{X}: X \rightarrow \mathscr{F}_{\mathfrak{W} T} T^{\Sigma}(X)
$$

(respecting substitutions) uniquely extends to an interpretation by structural recursion on $\Theta$-terms where maps $h_{X}$ and $b_{X}$ define the inductive and base cases respectively. Moreover, these maps can be easily given e.g. as equations, as we will show in Section 4

\section{Applications and Examples}

In this Section we illustrate how existing systems and specification formats are covered by the proposed WFSOS format. In particular, to illustrate the use of the format we give a detailed WFSOS specification for PEPA [12,13]. Then, instead of describing other specific cases, we devote the rest of the section to comparing WFSOS with some existing GSOS formats for systems expressible in the ULTraS framework.

\subsection{WFSOS for PEPA}

In PEPA [12,13], processes are terms over the grammar:

$$
P::=(a, r) \cdot P|P+P| P \underset{L}{\bigotimes} P|P \backslash L| \mathrm{x}
$$

where $a$ ranges over a fixed set of labels $A, L$ over subsets of $A, r$ over $\mathbb{R}^{+}$, and x over a fixed set of process constants symbols $X$. Process symbols are associated to processes by terms of the form $\mathrm{x} \triangleq P$ such that there is exactly one term for every symbol $\mathrm{x} \in X$. The semantics of process terms is usually defined by the inference rules in Figure 1 where $a \in A, r, r_{1}, r_{2}, R \in \mathbb{R}^{+}$(passive rates are omitted for simplicity) and $R$ depends only on $r_{1}, r_{2}$ and the intended meaning of synchronisation. For instance, in 


$$
\begin{aligned}
& \underset{(a, r) . P \stackrel{a, r}{\longrightarrow} P}{\frac{P_{1} \stackrel{a, r}{\longrightarrow} Q}{P_{1}+P_{2} \stackrel{a, r}{\longrightarrow} Q}} \quad \frac{P_{2} \stackrel{a, r}{\longrightarrow} Q}{P_{1}+P_{2} \stackrel{a, r}{\longrightarrow} Q} \quad \frac{P \stackrel{a, r}{\longrightarrow} Q}{\mathrm{x} \stackrel{a, r}{\longrightarrow} Q} \mathrm{x} \triangleq P \quad \frac{P \stackrel{a, r}{\longrightarrow} Q}{P \backslash L \stackrel{a, r}{\longrightarrow} Q} a \notin L \quad \frac{P \stackrel{a, r}{\longrightarrow} Q}{P \backslash L} \stackrel{\stackrel{\tau, r}{\longrightarrow} Q}{\longrightarrow} Q \in L \\
& \frac{P_{1} \stackrel{a, r}{\longrightarrow} Q}{P_{1} \bigotimes_{L} P_{2} \stackrel{a, r}{\longrightarrow} Q \bigotimes_{L} P_{2}} a \notin L \quad \frac{P_{2} \stackrel{a, r}{\longrightarrow} Q}{P_{1} \bigotimes_{L} P_{2} \stackrel{a, r}{\longrightarrow} P_{1} \bigotimes_{L} Q} a \notin L \quad \frac{P_{1} \stackrel{a, r_{1}}{\longrightarrow} Q_{1} P_{2} \stackrel{a, r_{2}}{\longrightarrow} Q_{2}}{P_{1} \bigotimes_{L} P_{2} \stackrel{a, R}{\longrightarrow} Q_{1} \bigotimes_{L} Q_{2}} a \in L
\end{aligned}
$$

Figure 1: Structural operational semantics for PEPA.

applications to performance evaluation [12], rates model times and $R$ is defined by the minimal rate law:

$$
R=\frac{r_{1}}{r_{a}\left(P_{1}\right)} \cdot \frac{r_{2}}{r_{a}\left(P_{2}\right)} \cdot \min \left(r_{a}\left(P_{1}\right), r_{a}\left(P_{2}\right)\right)
$$

where $r_{a}$ denotes the apparent rate of $a$. In applications to systems biology [7], rates model molecules concentrations and $R$ is defined by the multiplicative law: $R=r_{1} \cdot r_{2}$.

PEPA can be characterized by a specification in the WFSOS format where the process signature $\Sigma$ is the same as (2) and weights are drawn from the monoid of positive real numbers under addition extended with the $+\infty$ element (only for technical reasons connected with the (-) and process variablesdifferently from other stochastic process algebras like EMPA [4], PEPA does not allow instantaneous actions, i.e. with rate $+\infty$ ). The intermediate language of weight terms is expressed by the grammar:

$$
\theta::=\varnothing\left|\diamond_{r}(\theta)\right| \theta_{1} \oplus \theta_{2}\left|\theta_{1} \|_{L} \theta_{2}\right| \xi \mid P
$$

where $r \in \mathbb{R}_{0}^{+}, L \subseteq A, \xi$ range over weight functions, and $P$ over processes. Note that the grammar is untyped since it describes the terms freely generated by the signature $\Theta=\left\{\varnothing, \diamond_{r}, \oplus, \|_{L}\right\}$, over weight function variables and processes. The meaning of these operators is given formally below by the definition of the interpretation (-D) which is introduced (by structural recursion on $\Theta$-terms) alongside WFSOS rules for presentation convenience. Intuitively $\varnothing$ is the constantly 0 function, $\diamond_{r}$ reshapes its argument to have total weight $r, \oplus$ is the point-wise sum and $\|_{L}$ parallel composition e.g. by (3).

For each action $a \in A$ and rate $r \in \mathbb{R}^{+}$, a process $(a, r) . P$ presents exactly one $a$-labelled transition ending in the weight function assigning $r$ to the (sub)process denoted by the variable $P$ and 0 to everything else. Hence, the action axiom is expressed as follows:

$$
\overline{(a, r) . P \stackrel{a}{\longrightarrow} \nabla_{r}(P)} \quad\left(\nabla_{r}(\psi) D_{X}(t)= \begin{cases}\frac{r}{\mid\left\lceil|\psi\rangle_{X}\right\rceil \mid} & \text { if }(\psi\rangle_{X}(t) \neq 0 \\ 0 & \text { otherwise }\end{cases}\right.
$$

where $\diamond_{r}$ reshapes ${ }^{6}$ the function $(P)_{X}$ to equally distribute the weight $r$ over its support; in particular, since process variables will be interpreted as "Dirac-like" functions (see below) $\diamond_{r}(P)$ corresponds to the weight function assigning $r$ to $\sum$-term denoted by $P$.

Complementary to the action axiom, $(a, r) . P$ can not perform any action except for $a$ :

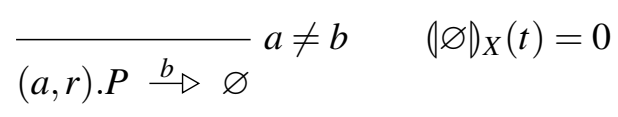

\footnotetext{
${ }^{6}$ Since the interpretation $(-)$ has to cover the language freely generated from $\Theta$, we can not use the (slightly more intuitive) "Dirac" operator $\delta_{r}(P)$ - where $P$ is restricted to be a process variable instead of a $\Theta$-term. Likewise, indexing $\delta_{r, P}$ over processes would break substitution independence i.e. naturality.
} 
This rule is required to obtain a functional ULTraS and is implicit in Figure 1 where disabled transitions are assumed with rate 0 as in any specification in the Stochastic GSOS or Weighted GSOS formats. Without this rule, transitions would have been disabled in the non-deterministic layer i.e. $(a, r) . P \quad b / \triangleright$.

Stochastic choice is resolved by the stochastic race, hence the rate of each competing transition is added point-wise as in Figure 1 (and in the SGSOS and WGSOS formats). This passage belongs to the stochastic layer of the behaviour (hence to the interpretation, in our setting) whereas the selection of which weight functions to combine is in the non-deterministic behaviour represented by the rules and, in particular, to the labelling. Therefore, the choice rules became:

$$
\frac{P_{1} \stackrel{a}{\longrightarrow} \varphi_{1} \quad P_{2} \stackrel{a}{\triangleright} \varphi_{2}}{P_{1}+P_{2} \stackrel{a}{\triangleright} \varphi_{1} \oplus \varphi_{2}} \quad(\psi \oplus \varphi)_{X}(t)=(\psi)_{X}(t)+(\varphi)_{X}(t)
$$

Likewise, process cooperation depends on the labels to select the weight function to be combined. This is done in the next two rules: one when the two processes cooperate, and the other when one process does not interact on the channel:

$$
\frac{P_{1} \stackrel{a}{\rightarrow} \varphi_{1} \quad P_{2} \stackrel{a}{\longrightarrow} \varphi_{2}}{P_{1} \underset{L}{\bigotimes} P_{2} \stackrel{a}{\longrightarrow} \varphi_{1} \|_{L} \varphi_{2}} a \in L \quad \frac{P_{1} \stackrel{a}{\longrightarrow} \varphi_{1} \quad P_{2} \stackrel{a}{\longrightarrow} \varphi_{2}}{P_{1} \underset{L}{\bigotimes} P_{2} \stackrel{a}{\longrightarrow}\left(\varphi_{1} \|_{L} P_{2}\right) \oplus\left(P_{1} \|_{L} \varphi_{2}\right)} a \notin L
$$

The combination step depends on the meaning of the cooperation, e.g. in the case of (3):

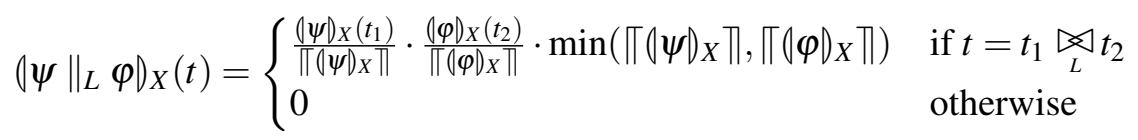

Each process is interpreted as a weight function over process terms. This is achieved by a Dirac-like function assigning $+\infty$ to the $\Sigma$-term composed by the aforementioned variable: $(P)_{X}(t)=+\infty$ if $P=t$, 0 otherwise. The infinite rate characterizes instantaneous actions as if all the mass is concentrated in the variable; e.g., in interactions based on the minimal rate law, processes are not consumed. For the same reason, if we were dealing with concentration rates and the multiplicative law, we would assign 1 to $P$.

The remaining rules for hiding and process symbol unfolding are straightforward?

$$
\frac{P \stackrel{a}{\rightarrow} \rho}{\mathrm{x} \stackrel{a}{\longrightarrow} \rho} \mathrm{x} \triangleq P \quad \frac{P \stackrel{a}{\longrightarrow} \varphi}{P \backslash L \stackrel{a}{\longrightarrow} \varphi} a \notin L \quad \frac{P \stackrel{a}{\longrightarrow} \varphi}{P \backslash L \stackrel{\tau}{\longrightarrow} \varphi} a \in L
$$

This completes the definition of (-) by structural recursion and hence the WFSOS specification of PEPA. It is easy to check that the induced ULTraS is functional and correspond to the stochastic system of PEPA processes; that bisimulations on it are stochastic bisimulations (and vice versa) and that bisimilarity is a congruence with respect to the process signature.

\subsection{Segala GSOS}

In [2, §5.3], Bartels proposed a GSOS specification format for Segala systems (whence Segala GSOS), i.e. ULTraS where weight functions are exactly probability distributions. In this Section, we recall Bartels' definition (with minor notational differences) and show how specifications in the aforementioned format can be translated in specifications for ULTraS.

\footnotetext{
${ }^{7}$ Specifications with equations, such as symbol unfolding rules, are handled thanks to the recent results proposed in 25].

${ }^{8}$ Specifications in Bartels' Segala GSOS format yield GSOS distributive laws for Segala systems but, to the best of authors knowledge, it still is an open problem whether every such distributive law arise from some specification in the Segala format.
} 
Definition 10 ( [2, §5.3]). A GSOS rule for Segala systems is an expression of the form:

$$
\frac{\left\{x_{i} \stackrel{a}{\rightarrow} \varphi_{i j}^{a}\right\}_{1 \leq i \leq n, a \in A_{i}, 1 \leq j \leq m_{i}^{a}}\left\{x_{i} \stackrel{b}{\rightarrow}\right\}_{1 \leq i \leq n, b \in B_{i}}\left\{\varphi_{i j}^{a} \Longrightarrow y_{k}\right\}_{1 \leq k \leq q}}{\mathrm{f}\left(x_{1}, \ldots, x_{n}\right) \stackrel{c}{\rightarrow} w_{1} \cdot t_{1}+\cdots+w_{m} \cdot t_{m}}
$$

where:

- $\mathrm{f}$ is an $n$-ary symbol from $\Sigma$;

- $X=\left\{x_{i} \mid 1 \leq i \leq n\right\}, Y=\left\{y_{k} \mid 1 \leq k \leq q\right\}$, and $V=\left\{\varphi_{i j}^{a} \mid 1 \leq i \leq n, a \in A_{i}, 1 \leq j \leq m_{i}^{a}\right\}$ are pairwise distinct process and probability distribution variables respectively;

- $a, b, c \in A$ are labels and $A_{i} \cap B_{i}=\emptyset$ for any $i \in\{1, \ldots, n\}$;

- $\left\{t_{i} \mid 1 \leq i \leq m\right\}$ are target terms on variables $X, Y$ and (possibly duplicated) $V$;

- $\left\{w_{i} \in(0,1] \mid 1 \leq i \leq m\right\}$ are weights associated to the target terms and s.t. $w_{1}+\cdots+w_{m}=1$;

A GSOS specification for Segala systems is a set of rules in the above format containing finitely many rules for any source symbol $\mathrm{f}$, conclusion label $c$ and trigger $\vec{C}$.

Segala GSOS specifications can be easily turned into WFSOS ones. The first two families of premises are translated straightforwardly to the corresponding ones in our format; the third can be turned into those of the form $\lceil\varphi\rceil \ni y$. Targets of transitions describe finite probability distributions and are evaluated to actual probability distributions by a fixed interpretation of a form similar to Definition 7 (although some care is needed to handle copies of probability variables).

Let $\tilde{V}$ be the set of "coloured" variables from $V$ where the colouring is used to distinguish duplicated variables (cf. [2, §5.3]). In practice, duplicated variables are expressed by adding "colouring" operators to $\Theta$; their number is finite and depends only on the set of rules since multiplicities are fixed and finite for rules in the above format. Given a substitution $v$ from $\tilde{V}$ to (finite) probability distributions over $T^{\Sigma}(X+Y)$, each $t_{i}$ is interpreted as the probability distribution:

$$
\tilde{t}_{i}(t) \triangleq \begin{cases}\prod_{k=1}^{\left|\tilde{V} \cap \operatorname{var}\left(t_{i}\right)\right|} v\left(\varphi_{k}\right)\left(t_{k}\right) & \text { if } t=t_{i}\left[\varphi_{k} / t_{k}\right] \text { for } t_{k} \in T^{\Sigma}(X+Y) \\ 0 & \text { otherwise }\end{cases}
$$

and each target term $w_{1} \cdot t_{1}+\cdots+w_{m} \cdot t_{m}$ is interpreted as the convex combination of each $\tilde{t}_{i}$.

\subsection{Weighted GSOS}

In [16], the authors propose a GSOS forma 9 for Weighted LTSs that is parametric in the commutative monoid $\mathfrak{W}$ and hence called $\mathfrak{W}-G S O S$. The format subsumes many known formats for systems expressible as WLTS: for instance, Stochastic GSOS specifications are in the $\mathbb{R}_{0}^{+}$-GSOS format and GSOS for LTS are in the $\mathbb{B}-G S O S$ format where $\mathbb{B}=(\{t t, f f\}, \vee, f f)$. In this Section, we recall Klin's definition and show how $\mathfrak{W}$-GSOS specification can be translated in WFSOS ones.

Definition 11 ( [16, Def. 13]). A W-GSOS rule is an expression of the form:

$$
\frac{\left\{x_{i} \stackrel{a}{\triangleleft} w_{a i}\right\}_{1 \leq i \leq n, a \in A_{i}}\left\{x_{i_{k}} \stackrel{b_{k}, u_{k}}{\triangleright} y_{k}\right\}_{1 \leq k \leq m}}{\mathrm{f}\left(x_{1}, \ldots, x_{n}\right) \stackrel{c, \beta\left(u_{1}, \ldots, u_{m}\right)}{\longrightarrow} t}
$$

where:

\footnotetext{
${ }^{9}$ Weighted GSOS specifications are proved to yield GSOS distributive laws for Weighted LTSs but it is currently an open question whether the format is also complete.
} 
- $\mathrm{f}$ is an $n$-ary symbol from $\Sigma$;

- $X=\left\{x_{i} \mid 1 \leq i \leq n\right\}, Y=\left\{y_{k} \mid 1 \leq k \leq m\right\}$ and $\left\{u_{k} \mid 1 \leq k \leq m\right\}$ are pairwise distinct process and weight variables;

- $\left\{w_{a i} \in \mathfrak{W} \mid 1 \leq i \leq n, a \in A_{i}\right\}$ are weight constants such that $w_{i_{k}} \neq 0$ for $1 \leq k \leq m$;

- $\beta: W^{m} \rightarrow W$ is a multiadditive function on $\mathfrak{W}$;

- $a, b, c \in A$ are labels and $A_{i} \subseteq A$ for $1 \leq i \leq n$;

- $t$ is a $\Sigma$-term such that $Y \subseteq \operatorname{var}(\mathrm{t}) \subseteq X \cup Y$;

A rule is triggered by a n-tuple $\vec{C}$ of enabled labels s.t. $A_{i}=C_{i} \subseteq$ A and by a family of weights $\left\{v_{a i} \mid 1 \leq\right.$ $\left.i \leq n, a \in C_{i}\right\}$ s.t. $w_{a i}=v_{a i} A \mathfrak{W}-G S O S$ specification is a set of rules in the above format such that there are only finitely many rules for the same source symbol, conclusion label and trigger.

Each rule describes the weight of $t$ in terms of weights assigned to each $y_{k}$ (i.e. $u_{k}$ ) occurring in it; if two rules share the same symbol, label, trigger and target then their contribute for $t$ is added.

The first step requires to make weight function explicit by premises like $x_{i} \stackrel{a}{\rightarrow} \varphi_{i}^{a}$ (since WLTS are functional ULTraS $m_{i}^{a}=1$ ). Then, each premise $x_{i} \stackrel{a}{\triangleleft} w_{a i}$ is translated into $\left\|\varphi_{i}^{a}\right\|=w_{a i}$. If $\mathfrak{W}$ is zerosumfree, the translation of a $\mathfrak{W}$-GSOS into a WFSOS is straightforward but, in general, it suffices to combine rules sharing the same source, label and trigger into a single one in the WFSOS format with the same source, label and trigger but whose target is a suitable weight term containing each $\beta$ and $t$ where every occurrence $y_{k}$ and $u_{k}$ is replaced with the corresponding function variable (i.e. $\varphi_{i_{k}}^{b_{k}}$ ), wrapped in a "colouring" operator to express multiplicities like in the case of Segala GSOS. In fact, the WFSOS specification for PEPA in Section 4.1 can be obtained from that given in [16] following this procedure.

\section{A coalgebraic presentation of ULTraS}

The aim of this section is to provide a brief coalgebraic characterization of ULTraSs and their bisimulation, and to prove that these bisimulations are congruence relations. These results are presented in Sections 5.2 and 5.3 respectively, and the general theory of abstract GSOS is briefly recalled in Section 5.1 (we refer the interested reader to [15,29]).

\subsection{Abstract GSOS}

In [29], Turi and Plotkin detailed an abstract presentation of well-behaved structural operational semantics for systems of various kinds. There syntax and behaviour of transition systems are modelled by algebras and coalgebras respectively. For instance, an (image-finite) LTS with labels in $A$ and states in $X$ is seen as a (successor) function $h: X \rightarrow\left(\mathscr{P}_{f} X\right)^{A}$ mapping each state $x$ to a function yielding, for each label $a$, the (finite) set of states reachable from $x$ via $a$-labelled transitions i.e. $\{y \mid x \stackrel{a}{\rightarrow} y\}$ :

$$
y \in h(x)(a) \Longleftrightarrow x \stackrel{a}{\rightarrow} y .
$$

Functions like $h$ are coalgebras for the (finite) labelled powerset functor $\left(\mathscr{P}_{f^{-}}\right)^{A}$ over the category of sets and functions Set. In general, state based transition systems can be viewed as B-coalgebrai.e. sets (carriers) enriched by functions (structures) like $h: X \rightarrow B X$ for some suitable covariant functor $B:$ Set $\rightarrow$ Set. The Set-endofunctor $B$ is often called behavioural since it encodes the computational behaviour characterizing the given kind of systems. A morphism from a $B$-coalgebra $h: X \rightarrow B X$ to $g: Y \rightarrow B Y$ is a function $f: X \rightarrow Y$ such that the coalgebra structure $h$ on $X$ is consistently mapped to the coalgebra structure $g$ on $Y$ i.e. $g \circ f=B f \circ h$. 


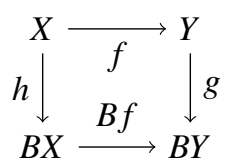

Therefore, $B$-coalgebras form the category $B$-Coalg of $B$-coalgebras and morphisms as above.

Two states $x, y \in X$ are said to be observationally equivalent with respect to the coalgebraic structure $h: X \rightarrow B X$ if they are equated by some coalgebraic morphism from $h$. Observational equivalences are generalized to two (or more) systems in the form of cocongruences i.e. as the pullbacks of morphisms extending to a cospan for the $B$-coalgebas structures associated with the given systems as pictured below.

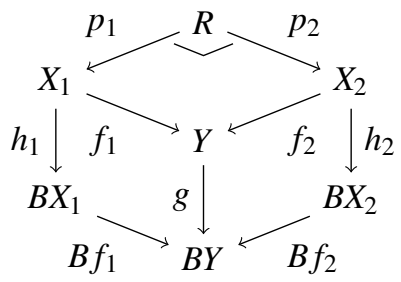

If the cospan $f_{1}, f_{2}$ is jointly epic, i.e. $j \circ f_{1}=k \circ f_{2} \Longrightarrow j=k$ for any $j, k: C \rightarrow Z$, (in general if $\left\{f_{i}\right\}$ is an epic sink, hence $\left\{p_{i}\right\}$ is a monic source) then the set $Y$ is isomorphic to the equivalence classes induced by $R$. We refer the interested reader to [26] for more information on the coalgebraic approach to process theory.

Dually, process syntax is modelled via algebras for endofunctors. Every algebraic signature $\Sigma$ defines an endofunctor $\Sigma X=\bigsqcup_{\mathrm{f} \in \Sigma} X^{\operatorname{ar}(\mathrm{f})}$ on Set such that every model for the signature is an algebra for the functor i.e. a set $X$ (carrier) together with a function $g: \Sigma X \rightarrow X$ (structure). Morphisms of $\Sigma$-algebras are functions satisfying the dual condition of (4). The set of $\Sigma$-terms with variables from a set $X$ is denoted by $T^{\Sigma} X$ and the set of ground ones admits an obvious $\Sigma$-algebra $a: \Sigma T^{\Sigma} \emptyset \rightarrow T^{\Sigma} \emptyset$ which is the initial $\Sigma$-algebra in the sense that for every other $\Sigma$-algebra $g$, there exists a unique morphism from $a$ to $g$ i.e. the inductive extension of the underlying function $f: T^{\Sigma} \emptyset \rightarrow X$. The construction $T^{\Sigma}$ is a functor, moreover, it is the monad freely generated by $\Sigma$.

In [29], Turi and Plotkin showed that structural operational specifications for LTSs in the well-known image finite GSOS format [5] are in a one-to-one correspondence with GSOS distributive laws i.e. natural transformations of the sort of:

$$
\lambda: \Sigma(\operatorname{Id} \times B) \Longrightarrow B T^{\Sigma} .
$$

Natural transformations like $\lambda$ contain the information needed to connect $\Sigma$-algebra structures and $B$ coalgebra structures over the same carrier set and capture the interplay between syntax and dynamics at the core of the SOS approach. These structures are called $\lambda$-bialgebras and are composed by a carrier $X$ endowed with a $\Sigma$-algebra $g$ and a $B$-coalgebra $h$ structure such that the diagram below commutes.

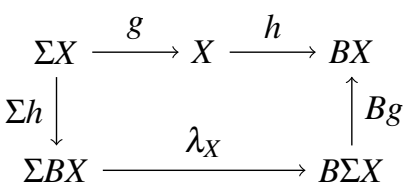

In particular, every $\lambda$-distributive law gives rise to a $B$-coalgebra structure over the set of ground $\Sigma$-terms $T^{\Sigma} \emptyset$ and to a $\Sigma$-algebra structure on the carrier of the final $B$-coalgebra. These two structures are part of the initial and final $\lambda$-bialgebra respectively and therefore, because the unique morphism from the former to the latter is both a $\Sigma$-algebra and a $B$-coalgebra morphism, observational equivalence on the system induced over $T^{\Sigma} \emptyset$ is a congruence with respect to the syntax $\Sigma$. 


\subsection{ULTraSs as coalgebras}

ULTraSs are shown to be coalgebras for Set endofunctors obtained by composing the functor $\left(\mathscr{P}_{f}(-)\right)^{A}$ of non-deterministic labelled transition systems with functors capturing the quantitative computational aspects. For every set $X$ we denoted by $\mathscr{F}_{\mathfrak{W}} X$ the set of finitely supported weight functions. Actually, this extends to a functor $\mathscr{F}_{\mathfrak{W}}:$ Set $\rightarrow$ Set over the category of sets, whose action on morphisms is given by (1) (it is easy to check that identities and compositions are preserved).

Proposition 5. For any $\mathfrak{W}$ and any A, coalgebras for $\left(\mathscr{P}_{f} \mathscr{F}_{\mathfrak{W}^{-}}\right)^{A}$ are in one-to-one correspondence with A-labelled image-finite $\mathfrak{W}$-ULTraSs.

Proof. Any image finite $\mathfrak{W}$-ULTraS $(X, A, \rightarrow)$ determines a coalgebra $(X, h)$ where, for any $x \in X$ and $a \in A: h(x)(a) \triangleq\{\rho \mid x \stackrel{a}{\triangleright} \rho\}$. Image finiteness guarantees that these sets are finite and that their elements are finitely supported weight functions from $X$ to the carrier of $\mathfrak{W}$. Then, it is easy to check that the correspondence is bijective.

The coalgebraic characterization is further justified by the corresponding treatment of bisimilarity:

Proposition 6. Let $\left(X, A, \rightarrow_{X}\right)$ and $\left(Y, A, \rightarrow_{Y}\right)$ be two image finite $\mathfrak{W}$-ULTraS. An equivalence relation between $X$ and $Y$ is a bisimulation (in the sense of Definition 3) iff it is the kernel relation of an epic sink for the coalgebras associated with the two systems.

Notice that we have used the behavioural equivalence (i.e. cocongruences) instead of Aczel-Mendler's coalgebraic bisimulation. The two notions coincide if the behavioural functor preserves weak pullbacks, but in general this is not the case for $\left(\mathscr{P}_{f} \mathscr{F}_{\mathfrak{W}^{-}}\right)^{A}$ (since $\mathscr{F}_{\mathfrak{W}}$ does not [16]). Actually, this property about $\mathscr{F}_{\mathfrak{W}}$ depends only on the underlying monoid, as it is equivalent to the row-column property [22] as stated by the following Lemma. This property can be easily checked, and more importantly holds for most monoids of interest.

Lemma 7. Coalgebraic bisimulation and behavioural equivalence coincide on every ULTraS iff the underlying monoid satisfies the row-column property i.e. for any two vectors $\left(w_{i}\right)_{i=1 \ldots n}\left(v_{i}\right)_{i=1 \ldots m}$ s.t. $\sum_{i=1}^{n} w_{i}=\sum_{i=1}^{m} v_{i}=s$ there exists a matrix $\left(u_{i j}\right)_{i=1 \ldots n, j=1 \ldots m}$ s.t. $\sum_{i=1}^{n} u_{i j}=v_{j}$ for each $j=1 \ldots$ m and $\sum_{j=1}^{m} u_{i j}=w_{i}$ for each $i=1 \ldots n$ as pictured aside.

\begin{tabular}{cccc|c}
$u_{1,1}$ & $u_{1,2}$ & $\cdots$ & $u_{1, n}$ & $w_{1}$ \\
$u_{2,1}$ & $u_{2,2}$ & $\cdots$ & $u_{2, n}$ & $w_{2}$ \\
$\vdots$ & $\vdots$ & $\ddots$ & $\vdots$ & $\vdots$ \\
$u_{m, 1}$ & $u_{m, 2}$ & $\cdots$ & $u_{m, n}$ & $w_{n}$ \\
\hline$v_{1}$ & $v_{2}$ & $\cdots$ & $v_{m}$ & $s$
\end{tabular}

Finally, we need the following technical result to unleash the general machinery of abstract GSOSs.

Proposition 8. The category of coalgebras for $\left(\mathscr{P}_{f} \mathscr{F}_{\mathfrak{W}^{-}}\right)^{A}$ has a final object.

\subsection{WFSOS as bialgebras}

In this subsection we prove the congruence result for bisimulations for ULTraSs induced by WFSOS specifications. This result is obtained by applying the abstract characterization of well-behaved structural operational semantics given by Turi and Plotkin in [29]. There syntax and behaviour of various kind of transition systems are modelled by algebras and coalgebras connected by suitable distributive laws describing the interplay between syntax and behaviour.

In the case of ULTraSs, the GSOS distributive laws are natural transformations of the form of:

$$
\lambda: \Sigma\left(-\times\left(\mathscr{P}_{f} \mathscr{F}_{\mathfrak{W}}(-)\right)^{A}\right) \Longrightarrow\left(\mathscr{P}_{f} \mathscr{F}_{\mathfrak{W}} T^{\Sigma}(-)\right)^{A}
$$

where $A$ is the set of labels, $\mathfrak{W}$ is the commutative monoid of weights, $\Sigma=\bigsqcup_{f \in \Sigma}(-)^{\operatorname{ar}(f)}$ is the syntactic endofunctor induced by the process signature $\Sigma$, and $T^{\Sigma}$ is the free monad for $\Sigma$. 
Every natural transformation $\lambda$ as above induces a coalgebra structure $h_{\lambda}$ (defined by structural recursion [29, Th. 5.1]) over ground $\Sigma$-terms as the only function $h_{\lambda}: T^{\Sigma} \emptyset \rightarrow\left(\mathscr{P}_{f} \mathscr{F}_{\mathfrak{W}}\left(T^{\Sigma} \emptyset\right)\right)^{A}$ such that:

$$
h_{\lambda} \circ a=\left(\mathscr{P}_{f} \mathscr{F}_{\mathfrak{W}} T^{\Sigma}\left(a^{\#}\right)\right)^{A} \circ \lambda_{X} \circ \Sigma\left\langle i d, h_{\lambda}\right\rangle
$$

where $a^{\#}: T^{\Sigma} T^{\Sigma} \emptyset \rightarrow T^{\Sigma} \emptyset$ is the inductive extension of $a$. Then, by general results from the bialgebraic framework (cf. [29, Cor. 7.3]), every behavioural equivalence on $h_{\lambda}$ is also a congruence on $T^{\Sigma} \emptyset$.

We can now provide the connection between WFSOS specifications and GSOS distributive laws for ULTraSs and between systems and coalgebras they induce over ground $\Sigma$-terms. Then, the results from abstract GSOS are transferred to the WFSOS specification format, completing the proof of Theorem 4.

Theorem 9 (Soundness). Every specification $\langle\mathscr{R},(-)\rangle$ yields a natural transformation $\lambda$ as in (5) such that $h_{\lambda}$ and the ULTraS induced by $\langle\mathscr{R},(-)\rangle$ coincide.

Corollary 10 (Congruence). Behavioural equivalence on the coalgebra over $T^{\Sigma} \emptyset$ induced by a specification $\langle\mathscr{R},(-D\rangle$ is a congruence with respect to the process signature $\Sigma$.

\section{Conclusions and future work}

In this paper we have presented a GSOS-style format for specifying non-deterministic systems with quantitative aspects. A specification in this format is composed by a set of rules for the derivation of judgements of the form $P \stackrel{a}{\longrightarrow} \psi$, where $\psi$ is a term of a specific signature, and an interpretation for these terms as weight functions. We have shown that a specification in this format defines an ULTraS system. The expressivity of this format has been shown by an example WFSOS specification for PEPA, and that WFSOS subsumes other formats such as WGSOS and Segala SOS. This format induces naturally a notion of bisimulation, which we have proved to be always a congruence. The proof of this result relies on a general categorical presentation of non-deterministic systems with quantitative aspects: we have shown that ULTraS systems are in one-to-one correspondence with coalgebras of a precise class of functors, parametric on the underlying weight structure. Taking advantage of Turi-Plotkin's bialgebraic framework, we have proved that the bisimulation induced by a WFSOS is always a congruence. This allows for compositional reasoning in quantitative settings (e.g., for ensuring performance properties).

Another consequence of this categorical characterization is that we can prove that there are $\lambda$ distributive laws which cannot be specified as WFSOS. In fact, we could define a more expressive format, but it would be quite more convolute and difficult to use. Hence, we preferred to adopt this simpler but still quite expressive format. The definition of a sound and complete format is left as future work.

In [19] the authors proposed the $n t \mu f \theta / n t \mu x \theta$ rule format for presenting Segala systems and such that the induced bisimilarity is a congruence. Because of the different expressivity of GSOS and (n)tree rules, it would be of interest to generalize this format to the wider range of behaviours covered by ULTraSs.

Although in this paper we have taken ULTraS systems as a reference, WFSOS can be interpreted in other meta-models, such as FuTS [18]. Like ULTraS, FuTS have state-to-function transitions, but admit several distinct domains for weight functions and hence can be read as "composing in parallel" distinct behaviours. The results in this paper readily extend to FuTS since these systems can be seen as coalgebras for functors with a specific shape: $\left(\mathscr{P}_{f} \bigsqcup_{\mathfrak{W} \in \mathscr{W}} \mathscr{F}_{\mathfrak{W}}(-)\right)^{A}$ for $\mathscr{W}$ being the set of admitted weight domains. In this context it is easy to formulate compositionality results also for the framework for stochastic calculi proposed in [9]. A coalgebraic understanding of FuTS is presented in [18] but covers only the deterministic case (i.e. $\left.\left(\amalg_{\mathfrak{W} \in \mathscr{W}} \mathscr{F}_{\mathfrak{W}}(-)\right)^{A}\right)$, while ours is non-deterministic.

For sake of simplicity, we have characterized ULTraS systems using the functor $\mathscr{F}_{\mathfrak{W}}$. However, the results and definition presented here can be further generalized by taking generic behavioural functors 
in place of $\mathscr{F}_{\mathfrak{W}}$, thus considering systems that are coalgebras for functors of the form of $\left(\mathscr{P}_{f} B(-)\right)^{A}$. This would affect mostly the evaluation (-), while only minor changes to the rule format may be required in order to capture interactions between $\mathscr{P}_{f}$ and $B$ (like e.g. the total weight premises). This fact suggests to investigate systems with stratified (or "staged") behaviours via "stratified" specifications. We can develop general results at the abstract level of bialgebraic structural operational semantics, aiming to provide some modularity to the format. This line of research can be seen as complementary to Mosses' Modular SOS [23] and recent developments towards a GSOS equivalent [21] (which still are more "syntax bound" since the behavioural functor is not very changed by these compositions).

The categorical characterization of ULTraS systems paves the way for further interesting lines of research. One is to develop Hennessy-Milner style modal logics for quantitative systems at the generality level of the ULTraS framework. In fact, Klin has shown in [14] that HML and CCS are connected by a (contravariant) adjunction. A promising direction is to follow this connection between modal logic and SOS, taking advantage of the bialgebraic presentation of ULTraS provided in this paper. Another direction is to investigate the implications of recent developments in the coalgebraic understanding of internal moves for systems generalized by ULTraSs such as Weighted LTS [20] and Segala systems [6].

Acknowledgements We thank Rocco De Nicola and Daniel Gebler for useful discussion on the preliminary version of this paper. This work is partially supported by MIUR PRIN project 2010LHT4KM.

\section{References}

[1] Giorgio Bacci \& Marino Miculan (2012): Structural Operational Semantics for Continuous State Probabilistic Processes. In: Dirk Pattinson \& Lutz Schröder, editors: CMCS, Lecture Notes in Computer Science 7399, Springer, pp. 71-89. Available at http://dx.doi.org/10.1007/978-3-642-32784-1_5

[2] Falk Bartels (2004): On Generalised Coinduction and Probabilistic Specification Formats: Distributive Laws in Coalgebraic Modelling. Ph.D. thesis, CWI, Amsterdam.

[3] Marco Bernardo, Rocco De Nicola \& Michele Loreti (2013): A uniform framework for modeling nondeterministic, probabilistic, stochastic, or mixed processes and their behavioral equivalences. Information and Computation 225, pp. 29-82. Available at http://dx.doi.org/10.1016/j.ic.2013.02.004.

[4] Marco Bernardo \& Roberto Gorrieri (1998): A tutorial on EMPA: A theory of concurrent processes with nondeterminism, priorities, probabilities and time. Theoretical Computer Science 202(1-2), pp. 1-54. Available at http: //dx.doi.org/10.1016/S0304-3975(97)00127-8.

[5] Bard Bloom, Sorin Istrail \& Albert R. Meyer (1995): Bisimulation Can't be Traced. J. ACM 42(1), pp. 232-268. Available at http://doi.acm.org/10.1145/200836.200876.

[6] Tomasz Brengos (2014): On coalgebras with internal moves. CoRR abs/1402.6281. Available at http: //arxiv.org/abs/1402.6281.

[7] Muffy Calder, Stephen Gilmore \& Jane Hillston (2005): Automatically deriving ODEs from process algebra models of signalling pathways. In: Gordon Plotkin, editor: Proc. CMSB, pp. 204-215.

[8] Luca Cardelli \& Radu Mardare (2010): The Measurable Space of Stochastic Processes. In: Proc. QEST, IEEE Computer Society, pp. 171-180. Available at http://dx.doi.org/10.1109/QEST.2010.30.

[9] Rocco De Nicola, Diego Latella, Michele Loreti \& Mieke Massink (2013): A uniform definition of stochastic process calculi. ACM Computing Surveys 46(1), p. 5. Available at http://doi.acm.org/10.1145/ 2522968.2522973.

[10] Manfred Droste, Werner Kuich \& Heiko Vogler (2009): Handbook of weighted automata. Springer. Available at http://dx .doi .org/10.1007/978-3-642-01492-5. 
[11] Holger Hermanns, Ulrich Herzog \& Joost-Pieter Katoen (2002): Process algebra for performance evaluation. Theoretical Computer Science 274(1-2), pp. 43-87. Available at http://dx.doi.org/10.1016/ S0304-3975(00) 00305-4

[12] Jane Hillston (1996): A compositional approach to performance modelling. Cambridge University Press. Available at http://dx.doi.org/10.1017/CB09780511569951.

[13] Jane Hillston (2005): Process Algebras for Quantitative Analysis. In: LICS, IEEE Computer Society, pp. 239-248. Available at http://dx.doi.org/10.1109/LICS.2005.35.

[14] Bartek Klin (2009): Bialgebraic methods and modal logic in structural operational semantics. Information and Computation 207(2), pp. 237-257. Available at http://dx.doi.org/10.1016/j.ic.2007.10.006.

[15] Bartek Klin (2011): Bialgebras for structural operational semantics: An introduction. Theoretical Computer Science 412(38), pp. 5043-5069. Available at http://dx.doi.org/10.1016/j.tcs.2011.03.023

[16] Bartek Klin \& Vladimiro Sassone (2013): Structural operational semantics for stochastic and weighted transition systems. Information and Computation .

[17] Kim Guldstrand Larsen \& Arne Skou (1991): Bisimulation through Probabilistic Testing. Information and Computation 94(1), pp. 1-28. Available at http://dx.doi.org/10.1016/0890-5401(91)90030-6.

[18] Diego Latella, Mieke Massink \& Erik P. de Vink (2012): Bisimulation of Labeled State-to-Function Transition Systems of Stochastic Process Languages. In: Ulrike Golas \& Thomas Soboll, editors: Proc. ACCAT, Electronic Proceedings in Theoretical Computer Science 93, pp. 23-43. Available at http://dx.doi.org/ 10.4204/EPTCS.93.2.

[19] Matias David Lee, Daniel Gebler \& Pedro R. D’Argenio (2012): Tree rules in probabilistic transition system specifications with negative and quantitative premises. In: Bas Luttik \& Michel A. Reniers, editors: EXPRESS/SOS, EPTCS 89, pp. 115-130. Available at http://dx.doi.org/10.4204/EPTCS.89.9

[20] Marino Miculan \& Marco Peressotti (2013): Weak bisimulations for labelled transition systems weighted over semirings. CoRR abs/1310.4106. Available at http://arxiv.org/abs/1310.4106.

[21] Stefan Milius, Lawrence S. Moss \& Daniel Schwencke (2013): Abstract GSOS Rules and a Modular Treatment of Recursive Definitions. Logical Methods in Computer Science 9(3). Available at http: //dx.doi.org/10.2168/LMCS-9(3:28)2013, http://arxiv.org/abs/1307.2538.

[22] Lawrence S. Moss (1999): Coalgebraic logic. Annals of Pure and Applied Logic 96(1), pp. 277-317. Available at http://dx.doi.org/10.1016/S0168-0072(98)00042-6.

[23] Peter D. Mosses (1999): Foundations of Modular SOS. In: Miroslaw Kutylowski, Leszek Pacholski \& Tomasz Wierzbicki, editors: MFCS, Lecture Notes in Computer Science 1672, Springer, pp. 70-80. Available at http://dx.doi.org/10.1007/3-540-48340-3_7.

[24] Corrado Priami (1995): Stochastic pi-Calculus. Comput. J. 38(7), pp. 578-589. Available at http://dx. doi.org/10.1093/comjnl/38.7.578.

[25] Jurriaan Rot \& Marcello M. Bonsangue (2014): Combining Bialgebraic Semantics and Equations. In: Anca Muscholl, editor: FoSSaCS, Lecture Notes in Computer Science 8412, Springer, pp. 381-395. Available at http://dx.doi.org/10.1007/978-3-642-54830-7_25

[26] Jan J. M. M. Rutten (2000): Universal coalgebra: a theory of systems. Theoretical Computer Science 249(1), pp. 3-80. Available at http://dx.doi.org/10.1016/S0304-3975(00)00056-6.

[27] Roberto Segala \& Nancy A. Lynch (1995): Probabilistic Simulations for Probabilistic Processes. Nord. J. Comput. 2(2), pp. 250-273.

[28] Chris M. N. Tofts (1990): A Synchronous Calculus of Relative Frequency. In: Jos C. M. Baeten \& Jan Willem Klop, editors: CONCUR, Lecture Notes in Computer Science 458, Springer, pp. 467-480. Available at http://dx.doi.org/10.1007/BFb0039078

[29] Daniele Turi \& Gordon Plotkin (1997): Towards a mathematical operational semantics. In: Proc. LICS, IEEE Computer Society Press, pp. 280-291. 